\title{
ALTERNATIVAS PARA COMBATIR LA POBREZA EN EL MARCO DE LOS ODS
}

\author{
Gloria Nancy Ríos Yepes \\ Institución Universitaria Politécnico Grancolombiano \\ griosyep@poligran.edu.co \\ Marisol Salamanca Olmos \\ Institución Universitaria Politécnico Grancolombiano \\ msalamanca@poligran.edu.co
}

\begin{abstract}
Resumen
El objetivo de este artículo de reflexión es analizar la relación y el aporte que tienen los negocios inclusivos y la RSE vistos desde la cadena de valor, que vinculan a la base de la pirámide en los procesos productivos, que conlleven a la creación de valor y a la innovación como aporte a la disminución de la pobreza.
\end{abstract}

El presente documento se centra en una revisión teórica que permite hacer una aproximación al cumplimiento del ODS1Ffin de la pobreza, a partir de la revisión conceptual de la pobreza, gestión empresarial, responsabilidad social empresarial, innovación, negocios inclusivos y cadena de valor.

Resulta pertinente vincular la base de la pirámide a las cadenas de valor ya que se ofertan como una ventaja competitiva, en ese sentido, se analiza cómo contribuir a la disminución de la pobreza trabajando con la base de la pirámide donde el Estado, empresas privadas y la sociedad en conjunto sean incluyentes y orienten esfuerzos para combatirla desde la gestión empresarial. Las cadenas de valor facilitan que las empresas sean más incluyentes y sostenibles de forma que pueden contribuir a mejorar las condiciones de vida de la sociedad.

Este análisis se contrasta con los avances que en esta materia ha alcanzado América Latina, de manera que se pueda identificar si las cadenas de valor y la responsabilidad social han logrado contribuir con la disminución de la pobreza, esto con el propósito de avanzar en su estudio y se sugiere una fase práctica donde se puedan validar su aporte a la base de la pirámide con el fin de combatir la pobreza.

Palabras clave

Pobreza, cadenas de valor, innovación, negocios inclusivos, responsabilidad social. 


\section{Introducción}

Hablar de la pobreza y de alternativas para disminuirla son conceptos que se hacen cada vez más estrechos e inherentes y el análisis debe conllevar al entendimiento de la satisfacción de las necesidades básicas de la población a partir de los recursos escasos con los que se cuenta para la satisfacción de estas. Se ha documentado como la RSE, la inclusión, la innovación y las cadenas de valor pueden contribuir a la disminución de la pobreza, trabajados en conjunto.

El presente artículo pretende hacer un análisis de la necesidad de la incorporación de los pobres a la base de la pirámide, mediante las cadenas de valor y de esta manera poder contribuir con la disminución de la pobreza, para que esto sea posible se sugiere un papel activo por parte de las empresas quienes han venido involucrando estos conceptos al marco de la responsabilidad social.

El propósito de este artículo de reflexión es analizar la relación y el aporte que tienen los negocios inclusivos y la RSE vistos desde la cadena de valor, donde se vinculan a la base de la pirámide en los procesos productivos, que conlleven a la co-creación de valor y a la innovación como aporte a la disminución de la pobreza.

Bien lo explicaba Prahalad, "enfocar a los pobres como parte activa de un mercado y agente emprendedores", en ese sentido adquieren un reconocimiento en el mercado y las empresas apoyadas en la RSE deben desarrollar sus estrategias para involucrar en las diferentes etapas de producción, distribución, comercialización y consumo. 
Lo que se pretende es dejar una expectativa de la gama de posibilidades que se pueden trabajar para disminuir la pobreza al ser empresas inclusivas, innovadoras, gestoras sostenibles y con responsabilidad social; todo ayudado con las cadenas de valor que adicionan beneficios a todos los actores de una economía, en especial a los más vulnerables.

El documento se organiza de la siguiente forma: en primer lugar, se presenta una introducción conceptual de la pobreza, seguida por una revisión de los conceptos de Gestión Empresarial, Responsabilidad Social Empresarial, Innovación, Inclusión y ¿Cadenas de valor analizados para América Latina; finalmente se presentan las conclusiones donde se deja la posibilidad de realizar una etapa práctica para poder evidenciar si efectivamente el trabajo en conjunto de estas iniciativas contribuyen a la disminución de la pobreza.

\section{Marco teórico}

\section{Pobreza}

El tema de pobreza, hay que conceptualizarlo primero, es tan amplio que se tienen aproximaciones, se parte desde la claridad que varios autores le dan, y es la situación en la cual los seres humanos no pueden satisfacer sus necesidades básicas, como la alimentación, vivienda y vestido; es tan complejo su significado que se habla de pobreza absoluta, establece un mínimo de recursos al que un individuo, región o país debe tener acceso para que disfrute de una 
vida digna, con calidad; y la pobreza relativa, esta última dependiendo del contexto donde se desarrolle.

Para este análisis se toma el concepto que el Consejo Europeo define como pobres a:

«aquellas personas, familias o grupos cuyos recursos (materiales, culturales y sociales) son tan limitados que les hacen quedar excluidos del modo de vida mínimo aceptable en el Estado miembro en que habiten».

Según el libro Pobreza, corrupción, (in)seguridad jurídica, (2017) presenta dos conclusiones:

La primera es que la caracterización de la pobreza no puede hacerse siguiendo un único aspecto. La segunda es que la lucha contra la pobreza ha de ser sistémica y no centrada en un único elemento, aunque sea tan relevante como el de la renta.

Existen métodos multidimensionales para representar la pobreza; un método multidimensional para medir la pobreza que se ha desarrollado en los últimos años es el propuesto por el PNUD a través del Índice de Pobreza Humana, que toma en consideración tres aspectos centrales: tener una vida larga y saludable, tener determinado nivel de educación y tener un nivel de vida digno. El PNUD distingue, en sus estudios, entre los países en desarrollo y aquellos que forman parte de la OCDE.

El anterior aporte muestra nuevamente, según el objetivo de este escrito, que combatir la pobreza no se hace trabajando un único aspecto, ya sea la inclusión, la cadena de valor, entre otros, hay que incursionar en varias alternativas que apunten hacia el mismo horizonte, para combatirla hay que empezar a disminuirla; y que son realmente las empresas como instituciones privadas las que deben propender o trabajar por esto. 
Las causas, su medición no es objetivo de este trabajo, solo se menciona como complemento. Lo que sí es claro es que la pobreza representa necesidades básicas insatisfechas y provoca la exclusión social.

En el libro "Qué hacemos contra la pobreza", los autores proponen que la solución debe plantearse en tres niveles:

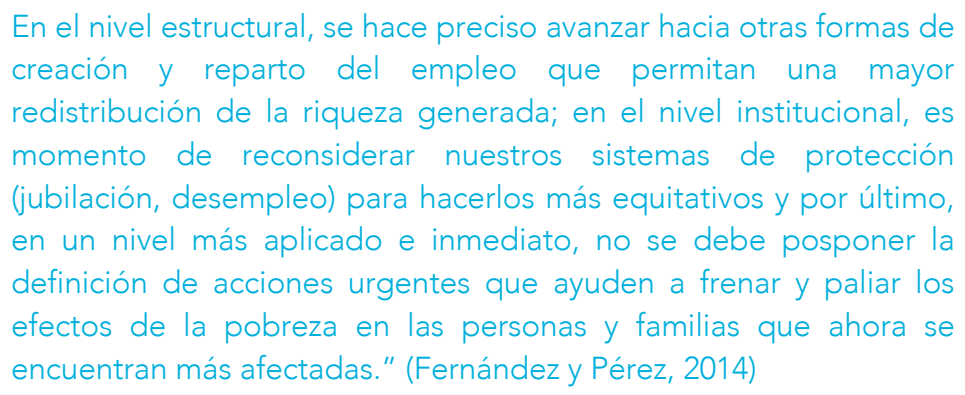

Es importante analizar el tema de la pobreza de forma estructural y unido a una distribución equitativa de la riqueza que se genera, así como lo que se gasta, por ejemplo, un individuo con una renta alta, podría gastar en restaurantes lo que un individuo de bajos ingresos gastaría en alimentarse durante un mes y más, si se trabaja de forma estructural, unido a estrategias en conjunto con las instituciones y con acciones urgentes e inmediatas, se podría tener otro panorama, puesto que se van posponiendo actividades en pro de disminuir la pobreza y siempre resulta algo más urgente e importante por resolver.

De acuerdo con lo anterior, se presenta una serie de alternativas que si se trabajan en conjunto pueden aminorar esta problemática que nos afecta a nivel mundial y que es interés de los gobiernos y de la sociedad disminuir tales indicadores; además, el autor menciona las instituciones en el nivel que se plantea para solucionar la pobreza, en lo cual se va a direccionar la siguiente parte de este escrito: 
Gestión empresarial

Con el concepto de pobreza claro, y de acuerdo con el panorama que se presenta para aportar a la solución de este problema por parte de las instituciones, se debe precisar en la gestión empresarial y la relación existente entre la Responsabilidad Social Empresarial y la sostenibilidad con relación a la Base de la Pirámide (BdP), Prahalad, en su libro La ortuna en la base de la pirámide lo direcciona hacia un mercado potencial que está en la base de la pirámide, se rescata de este contexto, el tema de la innovación, las personas más pobres del planeta tienen grandes capacidades empresariales y están ávidas por comprar.

Con innovación, sostenibilidad y trabajo colaborativo se puede ayudar a salir de la pobreza, entrelazado con la esencia de la economía, que tiene en cuenta la producción, distribución y consumo de forma eficiente en un mundo donde las necesidades son muchas y los recursos escasos. El autor adiciona: "Porque lo que usted sabe sobre los mercados de la base de la pirámide es erróneo. Un mundo de sorpresas, desde las pautas de consumo hasta la distribución y el marketing." (Prahalad, 2005)

La anterior posición en contexto con las empresas se estaría trabajando para proporcionar dignidad, capacitación y posibilidades, y no simplemente producir y producir. Tal como lo manifiesta el autor: "Las corporaciones y los empresarios de la base de la pirámide pueden obtener ganancias para todos, en el contexto de un nuevo capitalismo incluyente..." Un tema que va unido al objetivo de este trabajo, la inclusión, la cual se tomará más adelante. 
En ese sentido el conocimiento y la integración de los ODS a las políticas de desarrollo deben ser un ejercicio articulado que permita al Estado, al sector privado y a la población en general, contribuir con alternativas que conlleven al conocimiento, aplicación y logro de dichos objetivos de manera que se puedan alcanzar las metas establecidas y que en conjunto permitan mejorar las condiciones de pobreza, inequidad y una adecuada administración de los recursos, hasta aquí no se dejan solas las instituciones, se incluye el estado y la comunidad en general.

Es de vital importancia el papel que tienen las empresas como agente de cambio, ya que como se explica (Pacto Global de Naciones Unidas, 2015), las empresas en conjunto con el sector privado, los gobiernos y la sociedad tienen el mismo interés orientado a la estabilidad, la prosperidad, la paz y el desarrollo. Así se reitera la importancia que todos estos agentes adopten los ODS a su estructura y de esta manera si se consideraran como factores claves para contribuir con la disminución de la pobreza de la base de la pirámide.

\section{Responsabilidad Social Empresarial}

Al analizar las metas de los ODS en pro de mejorar las condiciones de vida del planeta y partiendo de una estrecha relación entre la Responsabilidad Social Empresarial y la sostenibilidad a la Base de la Pirámide $(\mathrm{BdP})$, se da como punto de partida la innovación de las empresas para generar más empleo como factor clave para disminuir la pobreza. Gracias a esta generación de empleo y por ende el 
aumento del consumo de las familias y empresas que activan la producción se trae el tema de la Quinta Revolución Industrial.

Según Peter Marsh en su libro La nueva Revolución Industrial: Consumidores, globalización y el final de la producción en masa, el autor señala que estamos viviendo una quinta etapa de la Revolución Industrial desde el año 2005 que duraría cuatro décadas o más.

\footnotetext{
"En su libro indica que esta nueva etapa se enfocará a la producción personalizada, en donde las características de un producto se adecuarán para un pequeño número de usuarios, o incluso una persona u organización, y aunque pareciera la antítesis de la producción masiva, habrá una mayor dispersión de la fabricación en todo el mundo, con numerosas conexiones transfronterizas y líneas de producción de menor escala que se ayudan mutuamente para lograr un mayor impacto global a través de los efectos de grupo." (Ramírez, 2019)
}

Se visualiza el fin de la producción en masa, donde con la globalización y los avances tecnológicos y científicos cada país puede hacer su aporte, de acuerdo con sus ventajas comparativas o absolutas, como lo presenta la masificación de su uso en todos los ámbitos de la economía mundial incluida la manufactura, a través de la gestión empresarial, teniendo como objetivo la responsabilidad social.

La responsabilidad social debe ser, entonces, inherente a la gestión de las empresas, la cual debe ser concebida dentro de los principios corporativos y aplicada en el hacer de cada empresa de manera colaborativa entre estas, de esta forma, lo expresa la autora del "Libro Responsabilidad Social Corporativa":

\footnotetext{
"La noción empresarial de responsabilidad social corporativa es un significante flotante que se llena de contenido en función del escenario de fuerzas ideológicas que determinan, en cada momento, cómo debe ser organizada la economía para controlar el poder de las empresas. Por lo tanto, la verdad en la que está el mundo de los negocios en relación con sus responsabilidades sociales depende de
} 
este juego de fuerzas y del sentido común que impere sobre lo que es razonable esperar de las corporaciones." (Gil, 2005)

Lo anterior está relacionado con lo que demostró John Nash, matemático y Premio Nobel de Economía 1994 con la teoría de juegos donde propendía por la terminación y la competencia entre los diferentes actores de los negocios e invitaba a la cooperación si se quería sacar adelante un proyecto y permitir su desarrollo; y quien más para aportar al desarrollo sostenible que las empresas, que impulsan la sostenibilidad del entorno en donde funcionan.

Así como lo plantea Paul Schweinzer, catedrático en el departamento de Economía de la Universidad de York, Inglaterra: "Cuando uno escoge algo, eso tiene un impacto en otras personas...". Si se opera de forma unida es más factible el logro de los ODS, se puede pensar en lograr estabilidad y crecimiento económico, que ayuda a disminuir la pobreza a nivel mundial. De ahí la importancia que las empresas no acojan los ODS solo en materia de sostenibilidad sino tomar estos compromisos como un principio de generación de ideas y oportunidades para crear negocios sostenibles, lo que permite crear empresas que perduren.

Las empresas tienen un interés común en especializarse en tareas específicas, intercambiar tecnología y aprender unas de otras. Esto se puede hacer de manera más fácil en el contexto de relaciones empresa a empresa a largo plazo.

Hablar de sostenibilidad y responsabilidad social desde un inicio ha reflejado que las empresas maximizaran su rentabilidad, sin pensar en una gestión social que podía aportar a la comunidad, es así como: 
Si hasta tiempos recientes existía un cierto consenso mayoritario acerca de que el «objetivo de la empresa» era maximizar el beneficio, ya desde finales de los años ochenta la persecución de este único objetivo se mostró endeble y una alternativa insuficiente e insostenible en un nuevo escenario. Dicho cambio paulatino fue reflejo de la propia complejidad y pluralidad progresiva de los mercados, donde entran en juego otros muchos agentes (stakeholders) y variables a considerar en la maximización de la función «objetivo» de la empresa. (Babé, 2016)

En la cimentación de este nuevo modelo de gestión sostenible, el propósito de las empresas se centra en generar valor, ya sea económico, social o ambiental. La RSE se convierte en un foco de interés y aporte a la sociedad.

Se habla de riesgos ambientales, cuando se requiere que los países optimicen la protección social y ambiental, y pueda hacer frente a un crecimiento desenfrenado del consumismo, debido a la globalización donde se olvida que estamos ante unos limitantes que son los recursos naturales:

\footnotetext{
“El sistema de producción y consumo debe medirse a partir de dos factores principales: los límites de nuestro planeta y una búsqueda insistente y permanente por la sostenibilidad. Ahora bien, el concepto de sostenibilidad para los empresarios de hoy se puede considerar a partir de la reflexión sobre la ineficacia de herramientas como la gestión ambiental, o los planteamientos iniciales que dieron origen a la ecoeficiencia..." (Boada, 2005, p.109)
}

De acuerdo con lo anterior se trata de encontrar un equilibrio entre lo que se produce y el impacto ambiental adicional que ha costado producirlo. Si se se ahorra energía, materiales y agua, habrá mayor producción y menos contaminación, lo cual tiene un efecto muy positivo en la lucha contra la pobreza. 
Lo presentado en los párrafos anteriores se puede visualizar en que si las empresas crecen, son sostenibles y rentables, además de cuidar el medio ambiente y generar valor agregado e innovar, no se verían afectadas por factores externos; no obstante, es importante cuidar y estar atento a lo que sucede en el entorno, en el caso de crisis, económicas, financieras, sociales o políticas, se observa:

Como tendencia se aprecia un correlato entre magnitud de pobreza y ciclos económicos, siendo los períodos recesivos y de crisis los que se asocian a un incremento en los niveles de pobreza de los países y, a la inversa, disminuyendo la tasa de pobreza en períodos de expansión económica. Esta tendencia general, admite algunas relativizaciones en el análisis empírico de América Latina. (Hardy, 2011)

Si se lograr una estabilidad y se sostiene en un mercado agresivo se estaría fortaleciendo en momentos de periodos de crisis, que como la historia nos lo ha mostrado son por un determinado tiempo, y es cuando hay que pensar en fortalecer las regiones y los factores productivos más vulnerables:

\footnotetext{
"Crisis y pobreza rural en américa latina. Aunque el mayor desafío en las zonas rurales es determinar los mecanismos de transmisión de la crisis en cada país, e identificar las políticas para contrarrestarlos, podemos concluir que en todos los contextos es necesario inducir un apoyo a las políticas agrarias, en particular las dirigidas a la pequeña agricultura y agricultura familiar con el fin de amortiguar el impacto de las crisis en las poblaciones más vulnerables y que esta población encuentre un trabajo y sustento en actividades productivas independientes (agropecuarias). Las estrategias que se desarrollen se presentan como una oportunidad para promover y renovar el medio rural y el fomento de nuevas oportunidades para iniciar diversificación y mejorar sus ingresos a futuro, mejorando a su vez los niveles de seguridad alimentaria." (Trivelli y Yancari, 2009, p.42)
} 
Otro punto que sugiere análisis son los objetivos de la RSE, entre los intereses económicos de la empresa y la verdadera compensación que tiene esta para la base de la pirámide, (Ancos, 2011) señala que frente a ese debate vienen surgiendo diferentes enfoques que finalmente apuntan a alivianar la pobreza desde perspectivas puramente sociales, al igual se viene señalando, la RSE no debe estar sujeta solo a la incorporación de los pobres a la cadena de valor como consumidores sino como productores que les signifique una mejora en su calidad de vida y su condición de pobreza.

Innovación

Cuando se habla de innovación, lo cual va directamente relacionado con el conocimiento, implica generar productos o servicios nuevos o producir los mismos de manera diferente, es decir, un cambio, un mejoramiento a un proceso a un bien o servicio en una empresa.

Todas las organizaciones están en continua búsqueda de innovar no solo para presentar un mejor producto o servicio, sino también para sostenerse en el tiempo y obtener rentabilidad, y como política de país para generar más empleo, después de cada época de crisis quedan grandes desafíos, es así como con el covid-19 se viene mostrando, nuevamente, que las empresas requieren más espacios digitales, avances tecnológicos; los clientes cada vez acceden más a través del comercio electrónico. 
Si las empresas generan valor agregado hay innovación, de lo contrario sería solo un cambio, crear sistemas de valor, como lo presenta el libro Cómo mejorar la logística de su empresa mediante la simulación:

"Es necesario enfocar, de manera innovadora y competitiva, la toma
de decisiones en la empresa, y reconsiderar el nuevo marco del
planteamiento estratégico de las operaciones, los conceptos de
producción ajustada (lean manufacturing), la logística como fuente
de ventaja competitiva y la necesidad de instrumentar herramientas
informatizadas para la planificación de la cadena de suministro".
(Ediciones Díaz de Santos, 2006)
El autor incluye la cadena de suministro como cadena de valor, el que se atreve a sacar un producto al mercado debe hacerle un seguimiento hasta que esté en manos de su consumidor y aún más, hasta asegurarse de tener un protocolo de manejo y control de residuos. La invitación es a integrar las diferentes cadenas en el proceso de producción y distribución, para prestar un mejor servicio, la experiencia y conocimiento que cada sector tiene aporta y suma en una economía, reiterando la importancia del trabajo colaborativo.

Al revisar diferentes textos sobre la sostenibilidad de las empresas, una de las formas de mantenerse en el mercado y generar empleo es aportando valor, de forma que: expresa:

"De forma que podemos apuntar que nuestro atraso relativo en
productividad tiene poco que ver con nuestro atraso tecnológico y
mucho con nuestro individualismo, que esteriliza capacidades
humanas. Empresas y universidades que crean espacios de
individualismo son tal vez un hecho diferencial propio que
deberíamos enmendar en el futuro." (Carballo, 2006)

Es interesante cómo el autor presenta varias alternativas para innovar porque no solo va relacionado con espacios digitales, informática y tecnología; estos son solo indicadores, si volvemos al significado de innovar como "generación de valor", encontramos que hay mucho por hacer en las empresas puesto que no son suficientes 
las políticas económicas con las que sean apoyadas, así como a los individuos sino que debe existir un planteamiento de invertir más en los empleados que utilizan el entorno digital, las herramientas, la tecnología y las materias primas. Adicionalmente, se debe educar para el trabajo en equipo y poder descifrar si la pobreza se da por falta de productividad, por falta de tecnología, o más tiene que ver con el individualismo.

Se presenta la innovación como elemento fundamental, máxime en países emergentes y en desarrollo que tienen la necesidad de disminuir los índices de pobreza. Hablar de innovación va ligado a la sostenibilidad, en los negocios se precisa sostenibilidad empresarial:

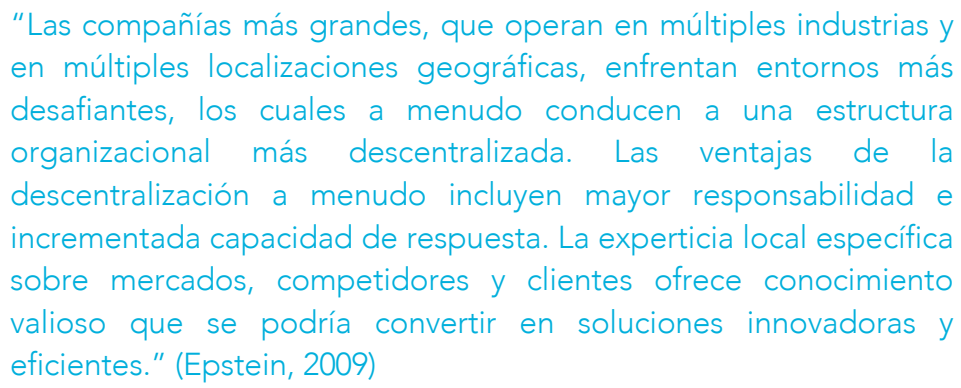

En este sentido se estaría ante un asunto de toma de decisiones autónomas, basado en operaciones más descentralizadas que permiten que los empresarios sean más innovadores, más creativos y la prueba "ensayo - error", viene de una forma repetitiva sin estar esperando la aprobación de un tercero, hay más experimentación, desarrollo de ideas nuevas y mayores ganancias para estas empresas. En el largo plazo esto significaría que las entidades descentralizadas realicen alianzas estratégicas y cada región se beneficie de los tratados de libre comercio existente, que permitan el logro de dichos objetivos. 
Lo anterior a largo plazo puede dar un resultado positivo, siempre y cuando las entidades descentralizadas realicen alianzas estratégicas y cada región acoja los TLC, que se convierten en una herramienta útil para alcanzar estos objetivos. Según Girón, 2016: “El artículo "Retos de América Latina: Agenda para el Desarrollo Sostenible y Negociaciones del siglo XXI", escrito por Ana María Álvarez, tiene como eje central la discusión sobre los múltiples procesos de integración vigentes y cómo éstos pueden coadyuvar a lograr las ambiciosas metas de desarrollo sostenible plasmadas en la "Agenda $2030^{\prime \prime}$

Los TLC reducen los costos de transacción y contribuyen a las economías de escala, pese a las dificultades presentadas por múltiples intereses, ya sea acuerdos bilaterales o mega regionales, ambos incluyen aspectos innovadores, fuera de los intereses políticos estos acuerdos asocian estándares laborales transfronterizos, contrataciones públicas, políticas de competencia, temas de interés mundial como el comercio y el medio ambiente, comercios electrónicos, propiedad intelectual, mejorar el acceso a los mercados para los productos agrícolas, entre otros.

La globalización y la integración mundial sugieren que las economías se adapten a estas nuevas tendencias de comercio, las cuales se convierten en una oportunidad para el sector empresarial, resulta importante empezar a ver en prospectiva cómo poder ser parte de esas cadenas de valor, ya que sin duda algunas son un factor de cambio importante que marca la tendencia del comercio mundial en diversos sectores. Sus procesos de fabricación sugieren diversas 
etapas e insumos a nivel mundial lo que puede llevar al aprovechamiento de esas ventajas competitivas, que se reflejen en un aumento de las exportaciones y crecimiento económico.

\section{Cadenas de Valor Mundial, CVM}

Según el informe sobre el desarrollo mundial 2020: comercio y desarrollo en la época de las cadenas de valor mundial, estas han nacido como la forma elevada de hacer negocios internacionales y disminuir el índice de pobreza, aunque tiene el foco en los países desarrollados, también es cierto que América Latina tiene oportunidad de ser partícipe.

Actualmente hay diferentes opiniones y estudios que muestran la importancia de las cadenas de valor mundiales para el desarrollo de un país, se encuentran ventajas y desventajas de este desarrollo activadas por las CVM, las del comercio permiten el crecimiento económico y reducen la pobreza, la productividad y el ingreso de las personas aumentó en países que hacían parte de estas cadenas de valor.

Al hablar de las cadenas de valor, también es importante reconocer algunas desventajas, como es el caso del valor agregado que generan y su distribución inequitativa, porque en todo caso se presentan riesgos ambientales, hay que pagar altos costos por un crecimiento acelerado, y en algunos casos tasas de desempleo.

Es claro que las cadenas de valor fomentan el crecimiento económico, generando empleo, aumentando la producción y el consumo y reduciendo la pobreza, no obstante, esto se puede ver opacado si no hay 
políticas inclusivas. Nuevamente retomamos este tema tan importante en este trabajo y por ende para disminuir la pobreza: la inclusión

En ese sentido, según Romero (2009) señala que las cadenas de valor globales permiten a las pymes en los países emergentes y en vía de desarrollo, grandes oportunidades de negocio y un medio para participar en los procesos de globalización, lo cual resulta positivo, pero a su vez sugiere amenazas. En cuanto a las oportunidades se destaca su crecimiento y desarrollo local, aumento en la tasa de empleo, acceso a mercados externos, procesos de mejora, eficiencia y competitividad.

Con relación a las amenazas, el mismo autor menciona que cuando las pymes mantienen una dependencia respecto a las corporaciones que la gobiernan, esto le sugiere una limitación para la obtención de ganancias elevadas, limitación en su capacidad de negociación, y estas mismas corporaciones pueden interferir en algunos procesos de actividades relacionadas como lo es el marketing.

A diferencia de la participación de las pymes en las cadenas de valor donde los suministros dependen de los intermediarios, estas tienen mayor libertad en los procesos de mejora (Romero, 2009), lo cual dependiendo de los objetivos de crecimiento y participación en el mercado, las pymes deben validar estos planteamientos en términos de oportunidades y amenazas, de manera que su inserción a estas cadenas de valor le resulte beneficioso en términos de aumento de sus ventas, participación en el mercado y racionamiento.

El Banco Interamericano de Desarrollo (2018), sugiere que aprovechar estas cadenas de valor a través de los suministros, puede 
ser una oportunidad para que la región diversifique sus exportaciones, y así, se podría entender como un factor de cambio importante para la región que centra su actividad económica de exportación en la extracción de recursos naturales, y por tanto es necesario aprovechar estas ventajas que ofrece el comercio mundial respecto a la posibilidad de poder tener una mejor participación, diversificar los productos e incorporar la innovación y la tecnología.

En torno a esto, el mismo informe presenta algunos indicadores económicos que muestran el atraso de América Latina respecto a otras regiones en cuanto a la participación en las cadenas de valor, por ejemplo, el índice de comercio intraindustrial, según datos de investigación del BID entre 1985 y 2010 aumentó un 35\% para los países de América Latina, mientras que para países de Asia y el Pacífico se duplico. Otro indicador que llama la atención son los vínculos de producción entre los países de la región, para este caso, el 50\% del valor agregado en Europa proviene de países de la región, 42\% para Asía y el Pacífico, mientras que en América Latina sugiere solo el 15\%. (Banco Interamericano de Desarrollo, 2018).

Frente a este panorama se convierte en un gran reto para la región poder ser parte de las cadenas de valor de comercio mundial, teniendo en cuenta una adecuada integración, mejor aprovechamiento de los tratados existentes, mejoras de la infraestructura vial de la región, pueden llevar a que A.L. se integre y fortalezca su crecimiento económico con el comercio a través de las cadenas de valor, potenciando la economía local y la estructura empresarial. 
El libro Cómo hacer de la cadena de suministro un centro de valor, apoya las operaciones y las cadenas de suministro como áreas creadoras de valor para la compañía y para el cliente. Para ello, desarrolla un modelo de cuatro pasos que permite crear una estrategia propia de estas áreas, completamente alineada con la estrategia de la compañía y con las expectativas del cliente. Este es el primer paso para transformar las cadenas de suministro en un centro de creación de valor. (Caja, C. Á.,2018).

La concepción de conectar lo local con lo global, podría ser una alternativa para vincular a esa cadena de valor a los campesinos, como bien lo menciona Harvard Business Review, (2009) "Cuando estas iniciativas integran pobres como productores, frecuentemente exportan su fuerza de trabajo a mercados consumidores consolidados, nacionales o internacionales, saltándose a los intermediarios y las barreras", desafortunadamente los campesinos agricultores quienes se encuentran al inicio de la cadena productiva entran a catalogarse como pobres, ya que no cuentan con la especialización, inversión, y terminan siendo los que asumen pérdidas, puesto que las ganancias terminan favoreciendo a los intermediarios.

Al hablar de RSE, o CVM, se cree que el tratamiento para América Latina debe ser distinto al resto del mundo, situación que se contradice, puesto que $A L$ con sus economías emergentes compite con cualquier país, cuenta con sus ventajas y bondades, es así como en el libro América latina piensa en américa latina, los problemas decisivos y los desafíos de la sociología histórica de estas regiones es lograr reponer a América Latina $(\mathrm{AL})$ en el centro de la atención en el 
campo de la reflexión teórica y de la formación de recursos humanos; hay que pensar en AL somos una sociedad capitalista y que estas sociedades se desarrollaron como sociedades de clase con la misma lógica que las sociedades europeas o de Norte América.

Negocios inclusivos

La incidencia que tienen los negocios inclusivos como alternativa para la disminución de la pobreza y contribución al cumplimiento de los ODS, se presenta la definición que sugiere el Social Enterprise Knowledge Network

\footnotetext{
"Un negocio inclusivo incorpora a los sectores de bajos ingresos, ya sea como consumidores, proveedores o distribuidores. Debe intentar transformar el statu quo, tener una motivación de negocios, conectar lo local y lo global, ser innovador y paciente, aprovechar los recursos locales y tener protagonistas no habituales." (Harvard Business Review, 2009)
}

Los negocios inclusivos desde su concepción teórica pueden contribuir a la disminución de la pobreza ya que al vincular a los más pobres a las cadenas de valor como proveedor, distribuidores o consumidores sin duda alguna puede permitir poner al servicio sus habilidades, conocimientos, adquirir un reconocimiento dentro de la misma como consumidor, y de esta manera que puedan ser aprovechados por las empresas mejorando sus condiciones e ingresos para que se permitan tener una mejor calidad de vida.

Incluir a la base de la pirámide a partir de los negocios inclusivos como bien lo menciona Harvard Business Review (2009), debe inicialmente incluir una "transformación del statu quo", esto es importante porque ya 
hay un reconocimiento dentro de la sociedad para todas aquellas familias de bajos ingresos, ya sea reconociendo sus motivaciones, deseos de compra y de cómo las empresas pueden brindar esos espacios para satisfacerlas, es entender cómo poner a disposición esos factores productivos de la empresa a su servicio, desde el punto de vista del consumidor, pero cuando se logran vincular a la cadena desde la producción o intermediario le puede representar mejores condiciones a su calidad de vida, a partir de mejores ingresos.

Aplicar adecuadamente el concepto de negocios inclusivos puede llevar a que los campesinos coloquen sus productos locales al mercado internacional, requiere esfuerzo por parte del Estado y organizaciones privadas sin intereses particulares que logren orientar estas políticas aprovechando las ventajas competitivas de cada región.

Es importante mencionar que cualquier proceso debe estar ligado a la innovación y a la generación de valor teniendo en cuenta las tendencias hacia donde se mueven los mercados, según el portal Euromonitor (2020), dentro de las tendencias para este año se puede mencionar: inclusión para todos, orgullo local en camino a ser global y cautívame en segundos.

Estas tendencias deberían ser conocidas por las empresas y los negocios, y adaptadas a las necesidades del consumidor para lo que se requiere un aporte de tiempo y de recursos, pero su desarrollo puede entenderse como una ventaja competitiva para la generación de valor no solo desde la cadena productiva sino hacia su consumidor final. 
Dentro de la tendencia inclusión para todos, se puede aportar a la base de la pirámide desde la innovación; un ejemplo de ello es el sector financiero que puede llegar a lugares apartados, y que significa un reconocimiento para las familias que puedan acceder a diversos productos, "Se debe procurar que los mecanismos de recurso en los mercados emergentes y las economías en desarrollo sean accesibles, eficientes y fáciles de usar para los diversos consumidores situados en la base de la pirámide" (Chapman \& Rafael, 2013). Esos mecanismos hacen referencias a que se pueda tener acceso a los servicios financieros en lugares remotos, que se puedan realizar transacciones y que las familias puedan hacer uso de estos servicios a través de entidades financieras, cooperativas, y diferentes canales.

Tal como lo expresa Ancos (2011) la dinámica de entender los negocios y su relacionamiento con el mercado ha cambiado, en ese sentido se hace importante identificar las dos nuevas tendencias que como factor de cambio generan un nuevo papel dentro de la sociedad inherente a la concepción de lo público y lo privado, el primero está relacionado con la demanda de la empresa y su compromiso con la responsabilidad social y el segundo relacionado con la creación de los negocios en la base de la pirámide, orientando su actividad y sus productos a favorecer a los más pobres de la sociedad.

No solo se debe hacer necesario el papel de Estado como garante de la satisfacción de esas necesidades básicas individuales, sino que las empresas como organizaciones independientes deben entrar a ser garantes a través de su estructura, con el objetivo de poder responder al mínimo de satisfacción de necesidades básicas, considerando que el 
bienestar individual puede conllevar a la prosperidad social y se puede llevar a cabo a partir de la relación de lo público con lo privado.

De acuerdo con la CEPAL (CEPAL, 2019) se estima que, en 2018, 182 millones de personas vivían en la pobreza y 63 millones en la extrema pobreza (CEPAL 2018), en este sentido como lo explica, se está muy lejos dar cumplimiento a la reducción de la pobreza, a pesar de que se han tenido menores tasas de desempleo, se ha mejorado la formalidad del trabajo, y los ingresos laborales medidos en salario mínimo. Desafortunadamente como lo muestra el informe, "La pobreza y la pobreza extrema" se presenta con mayor incidencia en niños, adolescentes y jóvenes, mujeres, áreas rurales, pueblos indígenas y afrodescendientes, características propias de la región marcada por la desigualdad, brechas de género, de ingresos, de educación y el acceso a servicios básicos.

Según CEPAL, en 2018, alrededor del 30,1\% de la población de América Latina estaba bajo la línea de pobreza, mientras que un 10,7\% se encontraba bajo el umbral de la pobreza extrema, lo que significa que, en dicho año, aproximadamente 185 millones de personas se encontraban en situación de pobreza y 66 millones de personas pertenecían a hogares con ingresos per cápita inferiores a la línea de pobreza extrema. Desde 2015 se ha venido observando en la región un aumento en los niveles de pobreza, y especialmente de pobreza extrema, aunque dicha tendencia perdió fuerza entre 2017 y 2018. Con todo, la pobreza total en 2018 superó en 2,3 puntos porcentuales a la registrada en 2014, lo que significa un aumento de alrededor de 21 millones de personas. La pobreza extrema creció 2,9 puntos 
porcentuales y aproximadamente 20 millones de personas entre 2014 y 2018. (CEPAL, 2019)

Según la $\mathrm{OCDE}$, las perspectivas económicas de América Latina instan a mejorar las capacidades domésticas y a adoptar una nueva visión de la cooperación internacional como facilitador para apoyar esos esfuerzos. A esto hay que apuntarle. (OCDE, 2019)

En el marco del cumplimiento de los ODS, y con el objetivo que su implementación contribuya a la disminución de la pobreza de la base de la pirámide, enmarcados a la responsabilidad social resulta importante las iniciativas que promuevan la empleabilidad desde las pequeñas y medianas empresas a través de la innovación y el desarrollo apuntándole a proyectos que transformen la sociedad y que sean sostenibles con el medio ambiente, garantizando el aprovechamiento y buen uso de los recursos naturales; en ese sentido la Agenda 2030 en el informe de la CEPAL, (CEPAL, 2019) destaca que la mejora de los indicadores sociales no depende solo de las políticas sociales que se promuevan desde el Estado y sus instituciones sino que se requiere una transformación productiva de toda la sociedad que apunte a "reducir la pobreza, promover la igualdad y proteger el medio ambiente".

Aún es necesario ampliar los esfuerzos, falta mucho por hacer, y en ese sentido como se plantea en la (CEPAL, 2019) para poder dar cumplimiento al ODS1, la reducción de la pobreza se debe dar a partir de la reducción de las desigualdades. En la medida que seamos una sociedad más inclusiva, y donde se apunte a un crecimiento económico sostenido basado en la educación y la inversión se lograría la reducción de la informalidad y una sociedad con más oportunidades, que no solo le 
apunte a la base de la pirámide sino a la disminución de las brechas anteriormente planteadas.

Cuando se habla de pobreza se piensa en cómo acabar con ella, de hecho, Naciones Unidas reporta que, en el 2.030, 167 millones de niños vivirán en la pobreza extrema si no hacemos algo para mejorar el acceso a la sanidad y la educación; por lo cual nos centramos en la inclusión, la innovación, las cadenas de valor y la sostenibilidad.

Hay que trabajar para que la competencia entre los diferentes actores de una economía busque la participación, compartir estrategias de desarrollo, con negocios inclusivos y colaborativos, haciendo énfasis en protagonismo de las empresas que impulsan la sostenibilidad.

\section{Conclusiones}

Cuando se empieza por conceptualizar qué es la pobreza y la relación que tiene con la gestión empresarial, la RSE, la innovación, cadenas de valor y negocios inclusivos se cree que es muy ambicioso pretender abarcarlos en un solo escrito; no obstante, al concluir este trabajo se muestra la estrecha relación que tienen con la pobreza, más bien, como pueden aportar a su disminución, si se trabajan en conjunto y si las empresas empiezan a incluirlas en su gestión y procesos administrativos, se puede evidenciar que una conlleva a la otra, veamos:

Con RSE las empresas están incluyendo trabajo colaborativo en su gestión, no es ajeno que las empresas enfrentan diversas dificultades 
de sostenibilidad por trabajar de forma aislada o por falta de recursos, se puede elegir una descentralización con estrategias que permita estar comunicados y apoyarse de forma colaborativa, en otras palabras, ejercer actividades de negocios en lo que se es bueno y decidir el nivel de control central versus autonomía de la unidad de negocios.

Es así como la RSE debe ser inherente a la gestión empresarial, de ahí la importancia que las empresas no acojan los ODS sólo en materia de sostenibilidad sino tomar estos compromisos como un principio de generación de ideas y oportunidades para crear negocios sostenibles, lo que permite crear empresas que perduren y generen empleo. Frente a ese aporte vienen surgiendo diferentes posibilidades que finalmente apuntan a alivianar la pobreza desde perspectivas sociales.

Al igual se deduce, la RSE no debe estar sujeta solo a la incorporación de los pobres a la cadena de valor como consumidores sino como productores y/o distribuidores, que les signifique una mejora en su calidad de vida y su condición de pobreza.

Es así como se plantea y se deja como expectativa, incluir de forma explícita y real a la gestión empresarial eficiente por medio de la innovación que atrae negocios inclusivos, aporta y genera valor a la gestión de las empresas, siempre y cuando su misión esté asociada con la RSE

El análisis presentado en el documento es un estudio exploratorio, de carácter cualitativo, que busca contrastar la relación y el aporte que tienen los negocios inclusivos y la RSE vistos desde la cadena de valor, donde se vinculan a la base de la pirámide en los procesos productivos, que conlleven a la cocreación de valor y a la innovación como aporte a la 
disminución de la pobreza. Además de contribuir con la literatura existente, este análisis se contrasta con los avances que en esta materia ha alcanzado América Latina, de forma que se pueda identificar si las cadenas de valor y la responsabilidad social han logrado contribuir con la disminución de la pobreza, con el propósito de avanzar en su estudio y poder identificar en próximas investigaciones una fase práctica donde se validen estas iniciativas.

Para concluir, el tema de la pobreza es tan amplio que no se puede hablar de una sola solución, hay que incluir una gama de alternativas, que trabajadas en conjunto y con responsabilidad pueden aportar a su disminución, tal es la gestión empresarial con una RSE coherente e inclusiva, mediante la innovación y la sostenibilidad.

Para el cumplimiento de los ODS se requiere innovación, creatividad, compromiso y como se presentó a lo largo de este trabajo, se requiere colaboración, un trabajo mancomunado entre el Estado y el sector empresarial, que representarían impactos sociales y ambientales compartidos. Es importante llevar esta investigación a una fase práctica que permita contrastar los planteamientos acá expuestos, con el apoyo desde la academia y el sector privado para contribuir al logro de los objetivos planteados.

\section{Referencias}

Acosta, Y., Ansaldi, W., y Giordano, V. (Eds.). (2015). América latina piensa en américa latina. https://ebookcentral-proquest-com.loginbiblio.poligran.edu.co

Agut Nieto, S., y Martín Hernández, P. (2007). Factores que dificultan el acceso de las mujeres 
a puestos de responsabilidad: una revisión teórica. Apuntes de Psicología, 25(2), 201214. http://copao.cop.es/files/contenidos/VOL25 2 7.pdf

Ancos, H. (2011). Negocios inclusivos y empleo en la base de la pirámide. En H. Ancos, Negocios inclusivos y empleo en la base de la pirámide (págs. 7-20). Madrid: Complutense S.A.

Babé, L. J., Cuesta, M. J., y Gomis, B. I. (2016). Gestión sostenible de las organizaciones: Modelo de responsabilidad. https://ebookcentral-proquestcom.loginbiblio.poligran.edu.co

Banco Interamericano de Desarrollo. (2018). Hecho en las Américas: Impulsando las cadenas de valor de nuestro hemisferio.

Boada, O. A. (2005). Negocios y sostenibilidad más allá de la gestión ambiental. https://ebookcentral-proquest-com.loginbiblio.poligran.edu.co

Caja, C. Á. (2018). Cómo hacer de la cadena de suministro un centro de valor. https://ebookcentral-proquest-com.loginbiblio.poligran.edu.co

Carballo, Roberto. Innovación y gestión del conocimiento, Ediciones Díaz de Santos, 2006.

CEPAL. (2019). Informe de avance cuatrienal sobre el progreso y los desafíos regionales de la Agenda 2030 para el Desarrollo Sostenible en América Latina y el Caribe.

CEPAL. (2019). Panorama Social de América Latina. https://repositorio.cepal.org/bitstream/handle/11362/44969/5/S1901133 es.pdf

Chapman, Megan y Rafael Mazer (2013), "Sistemas de recurso eficaces para los consumidores de servicios financieros de la base de la pirámide", Enfoques n. ${ }^{\circ}$ 90, Washington, DC: CGAP, diciembre

Cómo mejorar la logística de su empresa mediante la simulación, Ediciones Díaz de Santos,

Euromonitor International. (2020). Euromonitor revela las 10 principales tendencias globales de consumo para 2020 https://blog.euromonitor.com/euromonitor-revela-las-10principales- tendencias-globales-de-consumo-para-2020/

Fernández, M. G., Martínez, V. L., y Pérez, M. J. (2014). Qué hacemos contra la pobreza. https://ebookcentral-proquest-com.loginbiblio.poligran.edu.co

Fernández, Maillo, Guillermo, et al. Qué hacemos contra la pobreza, Ediciones Akal, 2014.

Girón, A. (2016). Problemas del Desarrollo. http://www.scielo.org.mx/scielo.php?script=sci arttext\&pid=S0301$\underline{70362016000300003}$

Hardy, C. (2011) De la pobreza a la desigualdad: políticas sociales post crisis, B - PENUD. ProQuestEbook Central. http://ebookcentral.proquest.com/lib/bibliopoligransp/detail.action?doclD=3203281.

Harvard Business Review. (2009). Negocios Inclusivos en América Latina. Harvard Business.https://www.researchgate.net/publication/228274326 Inclusive Businesses i n Latin A merica Spanish Version

La Casa del Libro. (2020). La fortuna en la base de la pirámide: como crear una vida digna y aumentar las opciones mediante el mercado. Obtenido de Marketing y publicidad:

OCDE et al. (2019), Perspectivas económicas de América Latina 2019: Desarrollo en transición, OECD Publishing, Paris, https://doi.org/10.1787/g2g9ff1a-es.

Pacto Global de Naciones Unidas. (2015). Avanzar en los objetivos de Desarrollo Sostenible 
apoyando la Paz: ¿Cómo pueden contribuir las empresas?

Pobreza, corrupción, (in)seguridad jurídica, Marcial Pons Ediciones Jurídicas y Sociales, 2017. ProQuestEbook Central. http://ebookcentral.proquest.com/lib/bibliopoligransp/detail.action?doclD=5486464. ProQuest Ebook Central. http://ebookcentral.proquest.com/lib/bibliopoligransp/detail.action?doclD=3221798. Ramírez, C, M. (2019). La quinta Revolución Industrial según Peter Marsh. Tecnológico de Monterrey. http://hdl.handle.net/11285/636038

Romero, L. I. (2009). PYMES y cadenas de valor globales: Implicaciones para la política industrial en las economías en desarrollo. En L. I. Romero, PYMES y cadenas de valor globales: Implicaciones para la política industrial en las economías en desarrollo (págs.

Trivelli, C., \& Yancari, J. (2009). Crisis y pobreza rural en américa latina. https://ebookcentralproquest-com.loginbiblio.poligran.edu.co

Zermeño, G. (2010). Pobreza y distribución del ingreso / julio boltvinik y enrique Hernández laos (reseña). estudios sociológicos. https://ebookcentral-proquestcom.loginbiblio.poligran.edu.co 